\title{
Contribution à l'étude des ondes longues irrotationnelles
}

\section{Contribution to the study of long irrotational waves}

\author{
PAR F. SERRE \\ INGÉNIEUR DOCTEUR

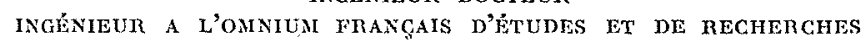

\begin{abstract}
Parallèle entre la théorie des ondes longues irrotationnelles en canal horizontal de faible profondeur et les écoulements permanents au voisinage de la hauteur critique. Exposé d'une méthode générale de calcul des ondes longues. Onde solitaire d'amplitude finie. Calcul du nombre de Froude correspondant à l'onde solitaire limite. Comparaison avec la thérie classique de Boussinesq et avec les résultats publiés par J.N. Hunt (La Houille Blanche, $\mathrm{n}^{\circ} 2,1955$ ).
\end{abstract}

La théorie des ondes longues irrotationnelles, dans un canal horizontal de faible profondeur, constitue un chapitre particulièrement intéressant de l'hydrodynamique à cause de sa relation avec les écoulements permanents au voisinage de la hauteur critique. On sait, en effet, qu'une onde de célérité constante $V$ peut être considérée comme stationnaire, en lui superposant un écoulement de vitesse constante et égale à - $\mathrm{V}$.

Considérons donc un écoulement uniforme de vitesse $\mathrm{V}$, correspondant à une hauteur d'eau $y_{0}$. Existe-t-il des solutions stables infiniment voisines de cette solution donnée?

La réponse est positive si le nombre de Froude de l'écoulement $\mathrm{F}=\mathrm{V} / \sqrt{\mathrm{g} y_{0}}$ est inférieur à 1 . La longueur d'onde $L$ de l'onde obtenue est donnée par l'équation (e) :

$$
\frac{\text { th } \sigma}{\sigma}=F^{2} \quad \text { où } \quad \sigma=\frac{\pi y_{0}}{\mathrm{~L}}
$$

Il n'en est plus de même lorsque $F$ est supérieur à 1 , l'équation $(e)$ n'ayant plus de racine réelle.

Si maintenant on envisage des ondes périodiques d'amplitude finie, on est conduit à la solution obtenue par Struick, le potentiel de l'écou-

\begin{abstract}
Parallel between the theory of long irrotational waves in shallow horizontal channels and permanent flows near the critical depth. Description of a general method of calculating long uaves. Solitary wave of finite amplitude. Method of calculating the Froude number corresponding to the limiting solitary wave. Comparison with Boussinesq's standard theory and with results published by J. N. HUNT (Houille Blanche, $\mathrm{n}^{\circ} 2$, 1955).
\end{abstract}

lement étant donné en fonction de l'amplitude relative par un développement en série.

Lorsque $F$ est petit, le rayon de convergence de cette série correspond à la houle limite (apparition d'un point anguleux à la crête). Il n'en est plus de même lorsque le nombre de Froude se rapproche de l'unité tout en restant inférieur à 1 .

La théorie de Struick ne peut donc ètre d'aucune utilité dans l'étude des ondes de translation au voisinage de la hauteur critique.

Nous nous proposons ici de développer une méthode générale de calcul des ondes longues en commençant par l'étude de l'onde solitaire, ce vocable désignant communément une intumescence se raccordant avec le niveau primitif à l'infini amont et aval.

\section{THEORIE DE L'ONDE SOLITAIRE}

Considérons l'écoulement uniforme torrentiel d'un fluide parfait sur un canal de fond horizontal, écoulement défini par sa profondeur $y_{0}$ et son nombre de Froude $\mathrm{F}=\mathrm{V} / \sqrt{\boldsymbol{g} \boldsymbol{y}_{0}}>1$.

L'onde solitaire de mêmes caractéristiques 
$\left(y_{0}, F\right)$ peut être considérée conme un écoulement irrotationnel permanent, se raccordant avec le précédent à l'infini amont et aval. Ces deux solutions ne sont pas infiniment voisines (l'amplitude de l'onde a une valeur finie liée à la valeur du nombre de Froude), sauf si $F_{--1}$ est un infiniment petit.

On est ainsi conduit à penser que l'écoulement est défini par une équation de la forme:

$$
\begin{aligned}
\frac{x}{y_{0}}+i \frac{y}{y_{0}}=\frac{\Phi}{v y_{0}} & +i \frac{\Psi}{\mathrm{V} y_{0}} \\
& +\mathrm{G}\left[\sigma \frac{(\Phi+i \Psi)}{\mathrm{V} y_{0}}, \sigma\right]
\end{aligned}
$$

dans laquelle :

$x$ et $y$ sont les coordonnées d'un point de l'écoulement, les axes ox, oy étant ceux de la figure 1 (l'origine $O$ est située sur le fond au droit de la crête);

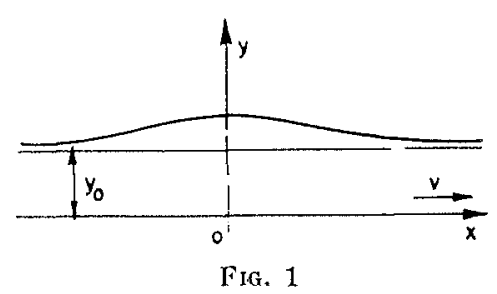

$\Phi$ et $\Psi$ sont la fonction potentielle et la fonction de courant, $\sigma$ est un paramètre sans dimension relié au carré du nombre de Froude par un développement en série de la forme :

$$
\begin{aligned}
\mathrm{F}^{2}=1+\alpha_{1} \sigma^{2}+\alpha_{2} \sigma^{4} & +\alpha_{n} \sigma^{2 n} \\
& +\ldots \quad \alpha_{1}>0
\end{aligned}
$$

absolument convergent pour $|\sigma|<\sigma_{1}$, et $\mathbf{G}(f, \sigma)$ est une fonction de la variable réelle $\sigma$ et de la variable complexe $f=\left(\sigma / V y_{0}\right)(\Phi+i \Psi)$ satisfaisant aux conditions suivantes :

C'est une fonction holomorphe de $f$ pour $|\sigma|<\sigma_{0}$ et $|\Psi|<v y_{0}$.

$\mathrm{G}(f, \sigma)$ tend uniformément vers zéro lorsque $\sigma$ tend vers zéro, autrement dit l'onde solitaire se réduit à un écoulement uniforme pour $F=1$.

$\mathrm{G}(f, \sigma)$ tend uniformément vers une limite constante réelle lorsque $\Phi$ augmente indéfiniment, autrement dit l'onde solitaire se raccorde à l'infini amont et aval avec l'écoulement uniforme $\left(y_{0}, F\right)$.

En dehors de ces conditions qui caractérisent l'onde solitaire, la fonction $\mathbf{G}(f, \sigma)$ doit être telle que l'écoulement plan irrotationnel, défini par (1), satisfasse aux conditions aux limites classiques des écoulements à surface libre.

Les fonctions harmoniques $x=x(\Phi, \Psi, \sigma)$, $y=y(\Phi, \Psi, \sigma)$, données par (1), sont liées' par les relations (3) :

$$
\frac{\partial x}{\partial \Phi}=\frac{\partial y}{\partial \Psi} \quad \frac{\partial y}{\partial \Phi}=\frac{\partial x}{\partial \Psi}
$$

qui permettent le passage de l'une à l'autre par des quadratures.

Portons notre attention sur la fonction

$$
y=y(\Phi, \Psi, \sigma)
$$

solution de l'équation de Laplace :

$$
\frac{\partial^{2} y}{\partial \Phi^{2}}+\frac{\partial^{2} y}{\partial \Psi^{2}}=0
$$

pour laquelle les conditions aux limites s'écrivent très simplement:

Condition au fond :

$$
\mathrm{Y}=0 \quad y=0
$$

Condition à la surface :

$\Psi^{2}=\mathrm{V} y_{0}, 2 g y+\frac{1}{\left(\frac{\partial y}{\partial \Psi}\right)^{2}+\left(\frac{\partial y}{\partial \Phi}\right)^{2}}=2 g y_{0}+\mathrm{V}^{2}(6)$

Ia constante de l'équation de Bernoulli a ici une valeur déterminée par suite de la condition de raccordement à l'infini avec l'écoulement uniforme.

Nous adopterons, dans la suite de l'exposé, des données sans dimensions, définies par les changements de fonction et de variable suivants, choisis de façon à mettre en évidence une méthode d'approximations successives relative au paramètre $\sigma$.

Nous poserons :

$$
\begin{aligned}
\xi & =\sigma \frac{x}{y_{0}} \\
\zeta & =\frac{y}{y_{0}} \\
z & =\frac{\sigma}{y_{0}}(x+i y)=\xi+i \sigma \zeta \\
\varphi & =\frac{\Phi}{V y_{0}} \\
\psi & =\frac{\Psi}{V y_{0}} \\
f & =\frac{\sigma}{V y_{0}}[\Phi+i \Psi]=\varphi+i \sigma \psi \\
\frac{d z}{d f}=v \frac{(u+i v)}{u^{2}+v^{2}}=v\left(\frac{\partial y}{\partial \Psi}+i \frac{\partial y}{\partial \Phi}\right) & =\frac{\partial \zeta}{\partial \psi}+i \sigma \frac{\partial \zeta}{\partial \varphi}
\end{aligned}
$$


Dans ces conditions, les relations (3) s'écrivent :

$$
\frac{\partial \zeta}{\partial \varphi}=\frac{\partial \zeta}{\partial \psi} \quad \frac{\partial \zeta}{\partial \psi}=-\sigma^{2} \frac{\partial \zeta}{\partial \varphi}
$$

$\zeta=\zeta(\varphi, \downarrow, \sigma)$ est la solution de l'équation :

$$
\frac{\partial^{2} \zeta}{\partial \psi^{2}}+\sigma^{2} \frac{\partial^{2} \zeta}{\partial \xi^{2}}=0
$$

qui satisfait aux conditions aux limites:

$$
\psi=0 \quad \zeta=0
$$

$\psi=1, \frac{\left.2(\zeta-1)\left[\left(\frac{\partial \zeta}{\partial \psi}\right)^{2}+\sigma^{2} \frac{\partial \zeta}{\partial \varphi}\right)^{2}\right]}{\left(\frac{\partial \zeta}{\partial \psi}\right)^{2}+\sigma^{2}\left(\frac{\partial \zeta}{\partial \varphi}\right)^{2}-1}=\mathrm{F}^{2}$

$F^{2}$ étant donné par le développement en série (2):

$$
\mathrm{F}^{2}=1+\alpha_{1} \sigma^{2}+\alpha_{2} \sigma^{4}+\ldots+\alpha_{n} \sigma^{2 n}+\ldots
$$

On voit ainsi que $\zeta=\zeta\left(0, \psi, \sigma^{2}\right)$ est une fonction de $\varphi, \psi$ et de $\sigma^{2}$ qui, pour $\sigma=0$, doit se réduire à :

$$
\zeta(0, \psi, 0)=\psi,
$$

l'onde solitaire se réduisant pour $\mathrm{F}=1$ à l'écoulement uniforme $\left(y_{0}, F\right)$. $\zeta$ est donc, pour $\sigma$ suffisamment petit, développable en série entière de $\sigma^{2}$, et on est conduit à rechercher une solution de $(4 a)$ de la forme:

$$
\zeta=\psi+\sigma^{2} \zeta_{1}(\varphi, \psi)+\ldots+\sigma^{2 n} \zeta_{n}(\varphi, \psi)+\ldots
$$

Laissant de côté pour le moment la condition $(6 a)$, nous nous donnerons avec BoussINESQ, la répartition des vitesses en tous les points du fond, en posant pour $\psi=0$ :

$$
\frac{\partial \xi}{\partial \varphi}\left(\rho, 0, \sigma^{2}\right)=\frac{\partial \zeta}{\partial \psi}\left(\varphi, 0, \sigma^{2}\right)=H\left(\varphi, \sigma^{2}\right)
$$

$H\left(\varphi, \sigma^{2}\right)$ étant une fonction paire de $\varphi$ (la répartition des vitesses doit évidemment être symétrique par rapport à la crête $o=0$ ) définie el continue, quel que soit p, pour $\sigma$ suifisamment petit. Pour satisfaire aux conditions écrites plus haut, caractérisant l'onde solitaire, il faut de plus que :

$$
H(\vartheta, 0) \equiv 1
$$

et que $\mathrm{H}\left(\varphi, \sigma^{2}\right)$ tende vers zéro lorsque augmente indéfiniment.

La fonction $\mathrm{H}\left(\varphi, \sigma^{2}\right)$ peut alors être définie par la série :

$$
\begin{aligned}
\mathbf{H}\left(\varphi, \sigma^{2}\right)=1+\sigma^{2} f_{1}(\varphi)+ & \sigma^{4} f_{2}(\varphi)+\ldots \\
& +\sigma^{2 n} f_{n}(\varphi)+\ldots
\end{aligned}
$$

uniformément convergente qucl que soit $\$$ pour - suffisamment petit, $f_{1}(\vartheta) \ldots f_{n}(\varphi) \ldots$ étant des fonctions paires de $\varphi$, définies et continues quel que soit $\varphi$ ainsi que leurs dérivées successives, et tendant vers zéro lorsque o augmente indéfiniment.

Ceci posé, en recherchant sous la forme (7) la solution de l'équation (4 a) qui satisfait aux données de Cavichy pour $\psi=0$ :

$$
\begin{gathered}
\zeta\left(\varphi, 0, \sigma^{2}\right)=0 \\
\frac{\partial \zeta}{\partial \psi}\left(\varphi, 0, \sigma^{2}\right)=\mathrm{H}\left(\varphi, \sigma^{2}\right)=1+\sigma^{2} f_{1}(\varphi)+\sigma^{4} f_{2}(\varphi)+\ldots+\sigma^{2 n} f_{n}(\varphi)+\ldots,
\end{gathered}
$$

on est conduit au développement :

$$
\begin{aligned}
\zeta=\psi+\sigma^{2} \psi f_{1}(\varphi)+\sigma^{4}\left[\psi f_{2}(\varphi)-\frac{\psi^{3}}{3 !} \frac{d^{2} f_{1}}{d \varphi^{2}}\right]+\sigma^{6}\left[\psi f_{3}(\varphi)-\frac{\psi^{3}}{3 !} \frac{d^{2} f_{2}}{d \varphi^{2}}+\frac{\psi^{3}}{5 !} \frac{d^{4} f_{1}}{d \varphi^{4}} \mid\right. \\
+\ldots+\sigma^{2 n+2}\left[\psi f_{n+1}(\varphi)-\frac{\psi^{3}}{3 !} \frac{d^{2} f_{n}}{d \varphi^{2}}+\ldots+(-1)^{n} \frac{\psi^{2 n+1}}{(2 n+1) !} \frac{d^{2 n} f_{1}}{d \varphi^{2 n}}\right]+\ldots
\end{aligned}
$$

supposé, pour le moment, uniformément convergent lorsque $\sigma$ est suffisamment petit et $|4| \leqslant 1$; on en déduit, par dérivation :

$$
\begin{aligned}
\frac{\partial \zeta}{\partial \varphi}=1+\sigma^{2} f_{1}(\varphi)+ & +\sigma^{4}\left[f_{2}(\vartheta)-\frac{\psi^{2}}{2 !} \frac{d^{2} f_{1}}{d \varphi^{2}}\right]+\sigma^{6}\left[f_{3}(\vartheta)-\frac{\psi^{2}}{2 !} \frac{d^{2} f_{2}}{d \vartheta^{2}}+\frac{\psi^{4}}{4 !} \frac{d^{4} f_{1}}{d \varphi^{4}} \mid\right. \\
& +\ldots+\sigma^{2 n+2}\left[f_{n+1}(\vartheta)-\frac{\psi^{2}}{2 !} \frac{d^{2} f_{n}}{d \vartheta_{j}^{2}}+\ldots+(-1)^{n} \frac{\psi^{2 n}}{(2 n) !} \frac{d^{2 n} f_{1}}{d \sigma^{2 n}}\right]+\ldots
\end{aligned}
$$




$$
\begin{aligned}
\frac{\partial \zeta}{\partial \varphi}=\sigma^{2} \psi \frac{d f_{1}}{d \varphi}+\sigma^{4} & {\left[\psi \frac{d f_{2}}{d \varphi}-\frac{\psi^{3}}{3 !} \frac{d^{3} f_{1}}{d \varphi^{3}}+\sigma^{6}\left[\psi \frac{d f_{3}}{d \varphi}-\frac{\psi^{3}}{3 !} \frac{d^{3} f_{2}}{d \varphi^{3}}+\frac{\psi^{5}}{5 !} \frac{d^{5} f_{1}}{d \varphi^{5}}\right]\right.} \\
& +\ldots+\sigma^{2 n+2}\left[\psi \frac{d f_{n+1}}{d \varphi}-\frac{\psi^{3}}{3 !} \frac{d^{3} f_{n}}{d \varphi^{3}}+\ldots+(-1)^{n} \frac{\psi^{2 n+1}}{(2 n+1)} \frac{d^{2 n+1} f_{1}}{d \varphi^{2 n+1}}\right]+\ldots
\end{aligned}
$$

Remarquons que $\zeta$ est donné par la partie imaginaire de :

$$
z=\int_{0}^{f} \mathrm{H}\left(f, \sigma^{2}\right) d f
$$

où :

car pour :

$$
z=\xi+i \sigma \zeta \quad \text { et } \quad f=\varphi+i \sigma \psi
$$

$$
\begin{gathered}
\psi=0 \\
\zeta=0 \quad \frac{d z}{d f}=\frac{\partial \xi}{\partial \varphi}=\frac{\partial \zeta}{\partial \varphi}=\mathrm{H}\left(\varphi, \sigma^{2}\right)
\end{gathered}
$$

Supposons que :

$$
\frac{d z}{d f}=\mathrm{H}\left(f, \sigma^{2}\right)=\frac{\partial \zeta}{\partial \varphi}+i \sigma \frac{\partial \zeta}{\partial \psi}
$$

soit développable en série de Taylor au voisinage de tout point du fond $\left(\psi=0, f=f_{0}=\varphi\right)$. Ceci exige que la fonction $H\left(\varphi, \sigma^{2}\right)$, qui représente le potentiel de l'écoulement, puisse être prolongée analytiquement au-dessous de la droite $\psi=0$, c'est-à-dire que $\psi=0$ soit un arc analytique régulier. La fonction $\mathrm{H}\left(\varphi, \sigma^{2}\right)$ doit donc, pour $\sigma$ suffisamment petit, être une fonction régulière de la variable réelle $\varphi$.

Dans ces conditions, on peut écrire, au voisinage de $f_{0}=\varphi$ :

$$
\mathrm{H}\left(f, \sigma^{2}\right)=\mathrm{H}\left(f_{0}, \sigma^{2}\right)+\left(f-f_{0}\right) \frac{\partial \mathrm{H}}{\partial f}\left(f_{0}, \sigma^{2}\right)+\ldots+\frac{\left(f-f_{0}\right)^{p}}{p !} \frac{\partial^{p} \mathrm{H}}{\partial f^{p}}\left(f_{0}, \sigma^{2}\right)+\ldots
$$

série absolument convergente pour ${ }_{n}$

$$
\left|f-f_{0}\right|=|\sigma \psi|<\mathrm{A}
$$

Or (14) s'écrit, compte tenu des' relations :

$$
\begin{gathered}
f-f_{0}=i \sigma \psi \quad \frac{\partial H}{\partial f}\left(f_{0}, \sigma^{2}\right)=\frac{\partial H}{\partial \varphi}\left(\varphi, \sigma^{2}\right): \\
\mathbf{H}\left(f, \sigma^{2}\right)=\frac{\partial \zeta}{\partial \psi}+i \sigma \frac{\partial \zeta}{\partial \varphi}=\mathbf{H}\left(\varphi, \sigma^{2}\right)+i \sigma \psi \frac{\partial H}{\partial \varphi}\left(\varphi, \sigma^{2}\right)+\ldots+\frac{(i \sigma \psi)^{n}}{p !} \frac{\partial^{p} H}{\partial \varphi^{p}}\left(\varphi, \sigma^{2}\right)+\ldots ;
\end{gathered}
$$

remplaçons, dans $(15), \mathrm{H}\left(\varphi, \sigma^{2}\right)$ par la série $(9)$ :

$$
\mathrm{H}\left(\varphi, \sigma^{2}\right)=1+\sigma^{2} f_{1}(\varphi)+\ldots+\sigma^{2 n} f_{n}(\varphi)+\ldots
$$

uniformément convergent quel que soit o pour $|\sigma|<\sigma_{1}$, et séparons les parties réelles et imaginaires, on retrouve les' développements (11) et (12).

En conclusion, si la série (9) est uniformément convergente quel que soit o pour $|\sigma|<\sigma_{1}$, on peut déterminer un nombre positif $\sigma_{0}<\sigma_{1}$ tel que les séries (10), (11) et (12) soient, pour $|\sigma|<\sigma_{0}$, uniformément convergentes dans tout le plan de l'écoulement, ce dernier étant défini par :

$$
-\infty<\varphi<+\infty \quad 0 \leqslant \psi \leqslant 1 .
$$

L'écoulement sera alors donné par la fonction analytique (13) :

$$
z=\int_{0}^{f} \mathrm{H}\left(f, \sigma^{2}\right) d f
$$

Ainsi se trouvent réunis tous les éléments nécessaires à la détermination de la fonction $\mathrm{H}\left(f, \sigma^{2}\right)$, qui doit être telle que soit satisfaite la cor Jition $(6 a)$ à la surface libre, 


\section{Détermination de $H\left(f, \sigma^{2}\right)$}

Le problème se pose de la manière suivante:

Déterminer les valeurs $\alpha_{1}, \alpha_{2} \ldots \alpha_{n}$ du développement (2) de $\mathrm{F}^{2}$ et les fonctions $f_{1}(\varphi), f_{2}(\varphi) \ldots$ $f_{n}(\varrho)$ du développement $(9)$ de $H\left(\varphi, \sigma^{2}\right)$ de facon à satisfaire identiquement, quels que soient $\sigma$ et $\varphi$, à la condition à la surface qui s'écrit, pour $\psi=1$ :

$\frac{2(\zeta-1)\left[\left(\frac{\partial \zeta}{\partial \psi}\right)^{2}+\sigma^{2}\left(\frac{\partial \zeta}{\partial \varphi}\right)^{2}\right]}{\left(\frac{\partial \zeta}{\partial \psi}\right)^{2}+\sigma^{2}\left(\frac{\partial \zeta}{\partial \vartheta}\right)^{2}-1}=F^{2}=1+\alpha_{1} \sigma^{2}$

$\zeta\left(\vartheta, 1, \sigma^{2}\right), \frac{\partial \zeta}{\partial \psi}, \frac{\partial \zeta}{\partial \rho}$ sont donnés par les séries (10), (11) et (12) dans lesquelles on fait $\psi=1$.

Rappelons que $f_{1}(\varphi), f_{2}(\varphi) \ldots f_{n}(\varphi)$ doivent en outre satisfaire aux conditions suivantes, désignées dans la suite par « conditions $C$ »

$1^{\circ}$ Elles doivent être des fonctions paires de $\varphi$, définies et continues quel que soit $\varphi$, ainsi que leurs' dérivées successives;

$2^{\circ}$ Elles doivent tendre vers zéro lorsque $९$ augmente indéfiniment par valeurs positives ou négatives;

$3^{\circ}$ La suite : $u_{n}=\left|f_{n}(\varphi) / f_{n-1}(\varphi)\right|$ doit être bornée supérieurement, quel que soit o, par un nombre A [convergence uniforme de la série (9)].

Ecrivons, pour $\psi=1$, l'identité : $\zeta, \partial \zeta / \partial \psi, \partial \zeta / \partial \varphi$ étant remplacés respectivement par les séries (10), (11) et (12) où l'on fait $\psi=1$.

On obtient, pour le premier membre de (16), une série ordonnée suivant les puissances de $\sigma^{2}$, dont tous les coefficients doivent être identiquement nuls.

On observe que cette série commence par un terme en $\sigma^{4}$. Cela tient au choix particulier du premier terme du développement de $F^{2}, \alpha_{0}=1$ (le coefficient $d u$ terme en $\sigma^{2} s^{\prime}$ écrirait, en posant $F^{2}=\alpha_{0}+\alpha_{1} \sigma^{2}+\ldots, 2 f_{1}(\varphi)\left(1-\alpha_{0}\right)$, ce qui entraîne, en dehors de la solution banale $f_{1}(\varphi) \equiv 0$, $*_{0}=1$ ). La première approximation ne déterminc donc pas $f_{1}(\varphi)$, mais donne simplement la valeur du premier terme du développement de $\mathrm{F}^{2}, x_{0}=1$. Ce résultat, qui constitue la formule classique de LAGRANGE, caractérise la singularité des écoulements au voisinage de la hauteur critique.

$\alpha_{0}$ étant ainsi pris égal à l'unité, on remarque que le coefficient de $\sigma^{*}$ est indépendant de $f_{2}(0)$ et, d'une façon générale, le coefficient de $\sigma^{2 n}$ est indépendant de $f_{n}(\varphi)$.

Ce résultat classique, que Boussineso mit le premier en évidence, nécessite, pour l'obtention de $f_{n}(\varphi)$, de recourir à l'approximation d'ordre $n+1$.

Nous déterminerons successivement $f_{1}(0)$ el $f_{2}(\varphi)$ par les équations de deuxième et lroisième approximation. Nous' montrerons ensuite, à l'aide d'un raisonnement par récurrence, que le calcul de $f_{r}(\varphi)$ se ramène à la résolution d'un système linéaire d'équations algébriques, la condition de compatibilité donnant le coefficient $\alpha_{n}$ du développement de $\mathrm{F}^{2}$.

$$
\begin{aligned}
2(\zeta-1)\left[\left(\frac{\partial \zeta}{\partial \psi}\right)^{2}+\sigma^{2}\left(\frac{\partial \zeta}{\partial \dot{\zeta}}\right)^{2}\right] \\
-\left[\left(\frac{\partial \zeta}{\partial \psi}\right)^{2}+\sigma^{2}\left(\frac{\partial \zeta}{\partial \varphi}\right)^{2}-1\right\rceil\left[1+\alpha_{1} \sigma^{2}+\alpha_{2} \sigma^{4}+\ldots+\alpha_{n} \sigma^{n}+\ldots\right]=0
\end{aligned}
$$

\section{EQUATION DE DEUXIEME APPROXIMATION}

On l'obtient en annulant le coefficient du terme en $\sigma^{t}$ dans le développement de (16). Il vient :

$$
\frac{1}{3} \frac{d^{2} f_{1}}{d \varphi^{2}}=\alpha_{1} f_{1}-\frac{3}{2} f_{1}^{2}
$$

équation du second ordre, dont une intégrale première s'écrit :

$$
\frac{1}{3}\left(\frac{d f_{1}}{d \varphi}\right)^{2}=\alpha_{1} f_{1}^{2}-f_{1}^{3}
$$

la constante d'intégration est prise égale à zéro, $f_{1}(\varphi)$ devant s'annuler asymptoliquement lorsque $\varphi$ augmente indéfinimenl.

Si $\alpha_{1}$ est positif [cette condition exprime que l'écoulement envisagé est torrentiel $\left[1^{2}>1\right]$, ce que nous supposerons rlans la suitel, l'équation (18) admet une intégrale, et une seule, consfituee par une fonction paire de o définie el continue, quel que soit $\varphi$; elle s'écrit :

$$
\begin{gathered}
\varphi= \pm \frac{1}{\sqrt{3}} \int_{a_{1}}^{f_{1}} \frac{d u}{u \sqrt{\alpha_{1}}-u} \\
f_{1}<u<\alpha_{1}
\end{gathered}
$$


intégrale qui s'exprime à l'aide des fonctions élémentaires sous la forme :

$$
f_{1}=\frac{\alpha_{1}}{\operatorname{ch}^{2}\left[\left(\sqrt{3 \alpha_{1}} / 2\right) \varphi\right]}
$$

Le paramètre $\alpha_{1}$ positif peut être choisi arbitrairement. L'onde solitaire de caractéristiques $\left(y_{0}, F\right)$ étant manifestement unique, on est conduit à penser que la solution envisagée, fonction a priori de $\alpha_{1}$ et $\sigma^{2}$, ne dépend en réalité que d'un seul paramètre $\lambda=\sqrt{\alpha_{1}} \sigma$. Ceci est vrai pour le premier ordre si on se rappelle qu'on a posé

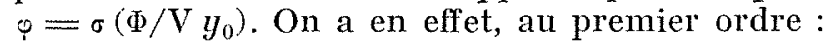

$$
\begin{gathered}
\mathrm{F}^{2}=1+\alpha_{1} \sigma^{2}=1+\lambda^{2} \quad \text { arbitraire susceptible de simplifier l'équation (19) } \\
\mathrm{H}\left(\varphi, \sigma^{2}\right)=1+\sigma^{2} f_{1}(\varphi)=1+\frac{\alpha_{1} \sigma^{2}}{\operatorname{ch}^{2}\left[(\sqrt{3} / 2) \sqrt{\alpha_{1} \sigma}\left(\Phi / \mathrm{V} y_{0}\right)\right]}=1+\frac{\lambda^{2}}{\operatorname{ch}^{2}\left[(\sqrt{3 / 2}) \lambda\left(\Phi / \mathrm{V} y_{0}\right)\right]}
\end{gathered}
$$

on devra de mème avoir pour l'ordre $n$ :

$$
f_{n}(\varphi)=\alpha_{1}^{n} g\left(\sqrt{\alpha_{1}} \varphi\right)
$$

Ce résultat diffère de celui auquel conduit la théorie classique des approximations linéaires. Dans celles-ci, en effet, la première approximation, dénommée par PoINcaré « équation aux variations $\gg$, est une équation linéaire et homogène, dont la solution est une fonction linéaire de la constante d'intégration. Il ne saurait évidemment en être de même pour le problème envisagé, où « l'équation aux variations » (18) n'est pas linéaire.

De facon à vérifier, pour les approximations ultérieures, l'hypothèse avancée plus haut, nous ne donnerons pas dès maintenant à $\alpha_{1}$ une valeur $\alpha_{n}=\mathrm{K}_{n} \alpha_{1}{ }^{n}, \mathrm{~K}_{n}$ étant indépendant de $\alpha_{1}$,

\section{EQUATION DE TROISIEME APPROXIMATION}

On l'obtient en annulant le coefficient de $\sigma^{\circ}$ dans le développement de (16). Il vient :

$$
\frac{1}{3} \frac{d^{2} f^{2}}{d \varphi^{2}}+f_{2}\left(3 f_{1}-\alpha_{1}\right)=\mathbf{R}_{2}\left[f_{1}, \frac{d^{2} f_{1}}{d \varphi^{2}},\left(\frac{d f_{1}}{d \varphi}\right)^{2}\right]
$$

où $\mathrm{R}_{\varepsilon}$ est une fonction déterminée de l'approximation précédente; si on y remplace $d^{2} f_{1} / d \varphi_{2}$ et $\left(d f_{1} / d \varphi\right)^{2}$ par leurs valeurs déduites de (17) et (18), on voit que $R_{2}$ est un polynôme du troisième degré en $f_{1}$ sans terme constant et on obtient, tous calculs faits :

$$
\begin{aligned}
\frac{1}{3} \frac{d^{2} f^{2}}{d \varphi^{2}}+f_{2}\left(3 f_{1}-\alpha_{1}\right) & =\left(\alpha_{2}-\frac{12}{10} \alpha_{1}{ }^{2}\right) f_{1}(\varphi) \\
+\frac{9}{2} \alpha_{1} f_{1}{ }^{2}-4 f_{1}^{3} & (21)
\end{aligned}
$$

$f_{1}(\varphi)$ étant donné par (19).

C'est une équation linéaire du second ordre; nous nous proposons de démontrer qu'elle admet une solution unique $f_{2}(\varphi)$ satisfaisant aux conditions $C$ décrites plus haut.

Remarquons que $d f_{1} / d \varphi$ est une solution particulière de l'équation sans second membre. En effet, la dérivation de (17) donne :

$$
\frac{1}{3} \frac{d^{2}}{d \varphi^{2}}\left[\frac{d f_{1}}{d \varphi}\right]+\frac{d f_{1}}{d \varphi}\left[3 f_{1}-\varkappa_{1}\right]=0
$$

L'intégration de (21) se ramène donc à deux quadratures en posant :

$$
f_{2}(\varphi)=\mathrm{K}(\varphi) \frac{d f_{1}}{d \varphi}
$$

(21) s'écrit, à l'aide du changement de fonction précédent et d'un facteur intégrant approprié :

$$
\begin{aligned}
& \frac{d}{d \varphi}\left[\frac{d \mathrm{~K}}{d \varphi} \times \frac{1}{3}\left(\frac{d f_{1}}{d \varphi}\right)^{2}\right] \\
& =\left[\left(\alpha_{2}-\frac{12}{10} \alpha_{1}^{2}\right) f_{1}+{ }_{2}^{9} \alpha_{1} f_{1}-4 f_{1}^{3}\right] \frac{d f_{1}}{d \varphi}
\end{aligned}
$$

ou, en intégrant :

$$
\begin{aligned}
\frac{d \mathrm{~K}}{d \varphi} \times \frac{1}{3}\left(\frac{d f_{1}}{d \varphi}\right)^{2}=\left(\alpha_{2}-\frac{12}{10} \alpha_{1}{ }^{2}\right) \frac{f_{1}^{2}}{2} \\
+\frac{3}{2} \alpha_{1} f_{1}^{3}-f_{1}^{4}
\end{aligned}
$$

La constante d'intégration est prise égale à zéro, $f_{2}(\varphi)$ devant s'annuler asymptotiquement lorsque $\$$ augmente indéfiniment.

$K(\varphi)$ s'obtient alors par une quadrature, en remplaçant $f_{1}(\varphi)$ et $d f_{1} / d \varphi$ par leur valeur déduite de (19) et en choisissant convenablement la constante d'intégration, de façon à obtenir 
pour $\mathrm{K}(\varphi)$ une fonction impaire

$$
f_{2}(\varphi)=\mathrm{K}(\varphi)\left(d f_{1} / d \varphi\right),
$$

$d f_{1} / d \varphi$ devant être paire.

Il vient, tous calculs faits :

$$
\begin{aligned}
& i_{2}(0)=\left(\alpha_{2}-\frac{12}{10} \alpha_{1}^{2}\right) \quad\left(\frac{1}{\operatorname{ch}^{2} \beta_{1} \varphi}-\beta_{1} \cdot \frac{\operatorname{sh} \beta_{1} \varphi}{\operatorname{ch}^{33} \beta_{1} \varphi}\right) \\
& +\frac{2 \alpha_{1}^{2}}{\operatorname{ch}^{4} \beta_{1} \rho}-\frac{\alpha_{1}^{2}}{\operatorname{ch}^{2} \beta_{1} \varphi}
\end{aligned}
$$

en posant pour simplifier $\beta_{1}=\left(\sqrt{3 \alpha_{1}}\right) / 2$,

Cette solution satisfait manifestement aux deux premières conditions $\mathrm{C}$, mais non à la troisième. En effet, le rapport $u_{2}=\left|f_{2}(\varphi) / f_{1}(\varphi)\right|$ n'est pas borné supérieurement, quel que soit $\varphi$, sauf si $\alpha_{2}=(12 / 10) \alpha_{1}^{2}$.

On est ainsi conduit à annuler le coefficient de $f_{1}$ dans le second membre de (21), $f_{2}(\varphi)$ prend alors la forme très simple:

$$
f_{2}(\varphi)=-\alpha_{1} f_{1}+2 f_{1}^{2}
$$

Montrons que, dans ces conditions, le calcul de $f_{2}(0)$ peut se ramener à la résolution d'un système linéaire d'équations algéhriques :

En effet, si on annule le coefficient de $f_{1}$ dans le second membre de (21) en posant $\alpha_{2.2}=(12 / 10) \alpha_{1}{ }^{2},(21)$ s'écrit :

$$
\begin{aligned}
\frac{1}{3} \frac{d^{2} f^{2}}{d \varphi^{2}} & +f_{2}\left(3 f_{1}-\alpha_{1}\right) \\
& =\frac{9}{2} \alpha_{1} f_{1}^{2}-4 f_{1}^{3}=\mathrm{A}_{21} \alpha_{1} f_{1}{ }^{2}+\mathrm{A}_{22} f_{1}^{3} ;
\end{aligned}
$$

cherchons pour $f_{2}(\varphi)$ une solution de la forme :

$$
f_{2}(\vartheta)=a_{21} \alpha_{1} f_{1}+a_{22} f_{1}^{2},
$$

dérivons deux fois, et tenons compte des expressions de $d^{2} f_{1} / d \varphi^{2}$ et $\left(d f_{1} / d \varphi\right)^{2}$ données par (17) et $(18)$; il vient :

$$
\begin{aligned}
\frac{1}{3} \frac{d^{2} f^{2}}{d \varphi^{2}} & +f_{2}\left(3 f_{1}-\alpha_{1}\right) \\
& \equiv\left(3 a_{22}+\frac{3}{2} a_{21}\right) \alpha_{1} f_{1}{ }^{2}-2 a_{22} f_{1}^{3}
\end{aligned}
$$

le coefficient du terme en $f_{1}$ étant identiquenent nul. En portant cette expression dans $(21 a)$ et en identifiant, on obtient:

$$
\left\{\begin{aligned}
-2 a_{22} & =\mathrm{A}_{22}=-4 \\
6 a_{22}+3 a_{21} & =2 \mathrm{~A}_{21}=9
\end{aligned}\right.
$$

d'où :

$$
a_{22}=2 \quad a_{21}=-1
$$

on retrouve ainsi très simplement l'expression (22).

\section{Forme GÊNÉrale DE $f_{\imath}(0)$}

Les résultats précédents permettent de dégager une méthode de récurrence, donnant la forme générale des approximations ultérieures.

Montrons que $\alpha_{n}$ et $f_{n}(0)$ sont respectivement de la forme :

$$
\begin{aligned}
\alpha_{n}= & \mathrm{K}_{n} \alpha_{1}{ }^{n} \\
f_{n}(\varphi)= & a_{n 1} \alpha_{1}^{n-1} f_{1}+\ldots \\
& \quad+a_{n p} \alpha_{1}^{n-p} f_{1}^{p}+\ldots+a_{n n} f_{1}^{n},
\end{aligned}
$$

où $K_{n}$ et $a_{n p}$ sont des nombres algébriques fractionnaires.

Cette forme étant exacte pour $n=1$ et $n=2$, il suffit de montrer que si elle est vraie jusqu'à l'ordre $n-1$ inclus, elle est vraie pour l'ordre $n$.

Dans ces conditions, $f_{n}(0)$ s'obtient en annulant dans le développement de (16) le coefficient $\mathrm{C}_{n}$ du terme en $\sigma^{2 n+2}$.

Ce coefficient est, nous le savons, indépendant de $f_{n+1}(0)$. En en isolant les termes contenant $f_{n}(\varphi)$ et $\alpha_{n}$, il vient :

$\frac{\mathrm{C}_{n}}{2} \equiv \frac{1}{3} \frac{d^{2} f_{n}}{d \varphi^{2}}+f_{n}\left(3 f_{1}-\alpha_{1}\right)-\alpha_{n} f_{1}-\mathrm{R}_{n}(\varphi)=0$

$R_{n}(\dot{\varphi})$ étant une fonction des approximations prćcédentes, c'est-à-dire de $f_{1}, f_{2} \ldots f_{n-1}(\varphi)$ et de leurs dérivées, ainsi que de $\alpha_{1}, \alpha_{2} \ldots \alpha_{n-1}$.

En se reportant au développement de (16) à l'aide des séries $(10),(11),(12)$ où l'on fait $\psi=1$, on montre sans difficulté que $R_{n}(\vartheta)$ est la somme du produit, par des nombres algébriques fractionnaires, de termes pouvant présenter les quatre formes suivantes:

$$
\begin{aligned}
& \frac{d^{2 p-2 s} f_{s+1}}{d \oplus^{2 p-2 s}} \mid \times \frac{d^{2 q-2 t} f_{1}}{d \varphi^{2 q-2 t}} \times \frac{d^{2 r-2 m} f_{m}}{d \varphi^{2 r-2 m}} \\
& \frac{d^{2 p-2 s} f_{s}}{d \varphi^{2 p-2 s}} \times \frac{d^{2 q t+1-2 t} f_{t}}{d \tau^{2} q+1-2 t} \times \frac{d^{2 r+1-2 m} f_{n t}}{d \varphi^{2 t+1-2 m}} \\
& q_{p+1} \times \frac{d^{2 t} t_{t}-2 t}{d p^{2} l_{1}-2 t} \times \frac{d^{2 r-2 m} \int_{m}}{d p^{2 r-2 m}} \\
& \alpha_{p} \times \frac{d^{2 q+1-2 t} \int_{1}}{d \varphi^{2 q+1-2 t}} \times \frac{d^{2 r+1-2 m} \int_{m}}{d \varphi^{2 r+1-2 m}}
\end{aligned}
$$


où $p, q, r, s, m, t$ sont des entiers quelconques, satisfaisant toutefois aux conditions suivantes :

$$
\begin{array}{lll} 
& p+q+r=n \\
o \leqslant s \leqslant p & o \leqslant t \leqslant q \quad o \leqslant m \leqslant r \\
p \leqslant n-2 & q \leqslant n-1 \quad m \leqslant n-1
\end{array}
$$

et avec les significations symboliques :

$$
\frac{d_{0} f_{l}}{d \varphi^{0}}=f_{t} \quad f_{0}(\varphi)=1 .
$$

Supposons maintenant que, jusqu'à l'ordre $n-1$ inclus, $\alpha_{p}$ et $f_{p}(\varphi)$ soient de la forme (23) et (24). On démontre facilement, en raisonnant par récurrence et compte tenu de (17) et (18), que $d^{2 q-2 t} f_{t} / d \varphi^{2 q-2 t}$ est, pour $q \geqslant t$, un polynôme $\left({ }^{*}\right)$ homogène et de degré $q$ des deux variables $\alpha_{1}$ et $f_{1}$, polynôme que nous désignerons par la notation :

$$
p_{q, t}\left(\alpha_{1}, f_{1}\right)
$$

avec :

$$
p_{q, t}\left(\alpha_{1}, 0\right)=0
$$

il s'ensuit que :

$$
\frac{d^{2 q+1-2 t} f_{t}}{d \varphi^{21+1-2 t}}=\frac{\partial p_{q . t}}{\partial f_{1}} \times \frac{d f_{1}}{d \varphi}
$$

est le produit par $d f_{1} / d{ }$, d'un polynòme homogène et de degré $q-1$.

On en déduit, compte tenu de (18), (23) et (25), que chaque terme de $R_{n}(\varphi)$, et par suite $R_{n}(\varphi)$ lui-même, est un polynôme homogène et de degré $(n+1)$, des deux variables $\alpha_{1}$ et $f_{1}$, s'annulant avec $f_{1}$ et dont tous les coefficients sont des nombres algébriques fractionnaires.

On peut donc écrire :

$$
\begin{aligned}
\mathrm{R}_{n}(\varphi)=\mathrm{A}_{n 0} \alpha_{1}^{n} f_{1}+\mathrm{A}_{n 1} \alpha_{1}^{n-1} f_{1}^{2} \\
+\mathrm{A}_{n p} \alpha_{1}^{n-p} f_{1}^{p+1}+\ldots+\mathrm{A}_{n n} f_{1}^{n+1}
\end{aligned}
$$

$f_{n}(\varphi)$ est donc solution de l'équation différentielle

$$
\begin{aligned}
& \frac{1}{3} \frac{d^{2} f_{n}}{d \varphi^{2}}+f_{n}\left(3 f_{1}-\alpha_{1}\right)=\left[\mathrm{A}_{n 0} \alpha_{1}^{n}+\alpha_{n}\right] f_{1} \\
& \quad+\mathrm{A}_{n 1} \alpha_{1}^{n-1} f_{1}^{2}+\ldots \\
& \quad+\mathrm{A}_{n p} \alpha_{1}^{n-p} f_{1}^{p+1}+\ldots+\mathrm{A}_{n n} f_{1}^{(n+1)}
\end{aligned}
$$

On démontre, comme pour (21), que cette équation admet une solution unique satisfaisant

(*) Les coefficients de ce polynôme sont, comme ceux de (24), des nombres algébriques fractionnaires. aux conditions $C$, si toutefois le coefficient de $f_{1}$ est nul, c'est-à-dire si :

$$
\alpha_{n}=-\Lambda_{n 0} \alpha_{1}^{n}
$$

condition qui donne le coeficient $\alpha_{n}$ du développement de $\mathrm{F}^{2}$ :

$$
\alpha_{n}=\mathrm{K}_{n} \alpha_{1}^{n} \quad \underline{\mathrm{K}_{n}=\cdots \mathrm{A}_{n 0}}
$$

Il reste à démontrer que cette solution est bien de la forme (24) annoncée plus haut.

En cherchant pour $f_{n}(\varphi)$ une solution de cette forme, on oblient, en dérivant deux fois et compte tenu de (17) et (18), l'expression de la quanlité :

$$
\frac{1}{3} \frac{d^{2} f_{n}}{d \wp^{2}}+f_{n}\left(3 f_{1}-\alpha_{1}\right)
$$

sous la forme d'un polynôme homogène et de degré $(n+1)$ des deux variables $\alpha_{1}$ et $f_{1}$, dans lequel les deux premiers termes du développement ordonné suivant les puissances décroissantes de $\alpha_{1}$ sont identiquement nuls.

En identifiant avec (26), on voit que, sous réserve que soit satisfaite l'égalité (23), $\alpha_{n}=\mathrm{K}_{n} \alpha_{1}{ }^{n}$, les coefficients $a_{n 1}, \ldots a_{n p} \ldots a_{n n}$ de l'expression (24) donnant $f_{n}(\varphi)$ sont donnés par le système linéaire :

$$
\left\{\begin{array}{c}
-a_{n n}\left(2 n^{2}+n-6\right)=2 \mathrm{~A}_{n n} \\
2 a_{n n}(n-1)(n+1) \\
-a_{n(n-1)}\left[2(n-1)^{2}+n-1-6\right]=2 \mathrm{~A}_{n(n-1)} \\
2 a_{n(p+1)} \times p(p+2) \\
-a_{n p}\left[2 p^{2}+p-6\right]=2 \mathrm{~A}_{n p} \\
6 a_{n 2}+3 a_{n 1}=2 \mathrm{~A}_{n 1}
\end{array}\right.
$$

d'où on tire successivement les valeurs de $a_{n n}$, $a_{n(n-1)} \ldots a_{n p} \ldots a_{n 1}$. Cette opération est toujours possible car, quel que soit l'entier $p$, la quantité $\left(2 p^{2}+p-6\right)$ est différente de zéro.

En définitive, $\alpha_{n}$ et $f_{n}(\varphi)$ ont bien la forme (23) et (24) annoncée plus haut.

\section{Calcul De $\mathrm{K}_{n}$}

Nous avons vu que $K_{n}=-A_{n 0}, A_{n 0}$ étant le coefficient de $\alpha_{1}^{n} f_{1}$ dans le développement de $\mathrm{R}_{n}(\varphi)$. La détermination de $A_{n 0}$ est relativement simple, car il suffit de considérer, dans le calcul 
de $\mathrm{R}_{n}(\varphi)$, les termes qui dépendent linéairement de $f_{1}, f_{2} \ldots f_{n-1}$ et de leurs dérivées. On obtient :

$$
\begin{aligned}
& \quad \alpha_{n-1}\left[f_{2}-\frac{1}{2 !} \frac{d^{2} f_{1}}{d \varphi^{2}}\right] \\
& +\alpha_{n-2}\left[f_{3}-\frac{1}{2 !} \frac{d^{2} f_{2}}{d \varphi^{2}}+\frac{1}{4 !} \frac{d^{4} f_{1}}{d \varphi^{4}}\right] \\
& +\ldots \\
& +\ldots \\
& +\alpha_{2}\left[f_{n-1}-\frac{1}{2 !} \frac{d^{2} f_{n-2}}{d \varphi^{2}}+\ldots+\frac{(-1)^{n-2}}{(2 n-4) !} \frac{d^{2 n-4} f_{1}}{d \varphi^{2 n-4}}\right] \\
& +\alpha_{1}\left[-\frac{1}{2 !} \frac{d^{2} f_{n-1}}{d \varphi^{2}}+\ldots+\frac{(-1)^{n-1}}{(2 n-2) !} \frac{d^{2 n-2} f_{1}}{d \varphi^{2 n-2}}\right] \\
& +\frac{1}{4 !} \frac{d^{4} f_{1-n}}{d \varphi^{4}}-\frac{1}{6 !} \frac{d^{6} f_{n-2}}{d \varphi^{6}}+\ldots+\frac{(-1)^{n}}{2 n !} \frac{d^{2 n} f_{1}}{d \varphi^{2 n}} \\
& -\frac{1}{5 !} \frac{d^{4} f_{1-n}}{d \varphi^{4}}+\frac{1}{7 !} \frac{d^{6} f_{n-2}}{d \varphi^{6}}-\ldots-\frac{(-1)^{n}}{(2 n+1) !} \frac{d^{2 n} f_{1}}{d \varphi^{2 n}}
\end{aligned}
$$

Or, on a :

$$
\begin{gathered}
f_{p}(p)=a_{p 1} \alpha_{1}^{p-1} f_{1}+\ldots\left(^{\star}\right) \\
\underline{a_{11}=1} \\
\frac{d^{2 q} f_{p-q}}{d \varphi^{2 q}}=a_{(p-q) 1} \times \alpha_{1}^{p-q-1}\left[\frac{d^{2 q} f_{1}}{d \varphi^{2 q}}+\ldots\right.
\end{gathered}
$$

et, compte tenu de (17):

$$
\frac{d^{2 q} f_{1}}{d \varphi^{2} q}=\left(3 \alpha_{1}\right)^{q} f_{1}+\ldots
$$

d'où :

$$
\frac{d^{2 q} f_{p-q}}{d \rho^{2 q}}=a_{(p-q)} \times 3^{q} \times \alpha_{1}^{p-1} f_{1}+\ldots
$$

posons de même :

$$
\alpha_{p}=\mathrm{K}_{p} \alpha_{1}^{p} \quad \mathrm{~K}_{1}=1 ;
$$

en portant ces diverses expressions dans (28), on obtient le premier terme du développement de $\mathrm{R}_{n}(\varphi)$ sous la forme $\mathrm{A}_{n 0} \alpha_{1}{ }^{n} f_{1}$.

Il vient, en définitive :

$$
\begin{aligned}
\mathrm{A}_{n 0}+\mathrm{K}_{n}=0= & \quad a_{(n-1) 1}\left[\mathrm{~K}_{2}-\frac{3}{2 !}+\frac{3^{2}}{4 !}-\frac{3^{2}}{5 !}\right] \\
& +a_{(n-2) 1}\left[\mathrm{~K}_{3}-\frac{3 \mathrm{~K}_{2}}{2 !}+\frac{3^{2}}{4 !}-\frac{3^{3}}{6 !}+\frac{3^{3}}{7 !}\right] \\
& +\ldots \\
+ & a_{21}\left[\mathrm{~K}_{n-1}-\frac{3}{2 !} \mathrm{K}_{n-2}+\ldots+\frac{(-1)^{n-2}}{(2 n-4) !} 3^{n-2}+\frac{(-1)^{n-1} 3^{n-1}}{(2 n-2) !}-\frac{(-1)^{n-1} 3^{n-1}}{(2 n-1) !}\right. \\
+ & \mathrm{K}_{n}-\frac{3}{2 !} \mathrm{K}_{n-1}+\ldots+\frac{(-1)^{n-2} 3^{n-2}}{(2 n-4) !} \mathrm{K}_{2}+\frac{(-1)^{n-1} 3^{n-1}}{(2 n-2) !}+\frac{(-1)^{n} 3^{n}}{(2 n) !}-\frac{(-1)^{n} 3^{n}}{(2 n+1) !}
\end{aligned}
$$

${ }^{\star}{ }^{*}$ Les termes suivants sont d’ordre supérieur à un, donc sans intérêt pour le calcul de $A_{n o}$. 
$\mathrm{Si}$, dans cette égalité, on fait successivement $n=2, n=3 \ldots$ de façon à calculer $K_{2}, K_{3}, \ldots$, on voit que $K_{n}$ est indépendant des coefficients $a_{(n-1) 1} \ldots a_{21}$, et donné par la suite définie par les relations :

$$
\left\{\begin{array}{l}
\mathrm{K}_{1}=1 \\
\mathrm{~K}_{2}-\frac{3}{2 !} \mathrm{K}_{1}+\frac{3^{2}}{4 !}-\frac{3^{2}}{5 !}=0 \\
\mathrm{~K}_{3}-\frac{3}{2 !} \mathrm{K}_{2}+\frac{3^{2}}{4 !} \mathrm{K}_{1}-\frac{3^{3}}{6 !}+\frac{3^{3}}{7 !}=0 \\
\ldots \ldots \ldots \ldots \ldots \ldots \ldots \ldots \ldots \ldots \ldots \ldots \ldots \ldots \ldots \ldots \ldots \ldots \ldots \\
\mathrm{K}_{n}-\frac{3}{2 !} \mathrm{K}_{n-1}+\ldots+\frac{(-1)^{n-2} 3^{n-2}}{(2 n-4) !} \mathrm{K}_{2} \\
\quad+\frac{(-1)^{n-1} 3^{n-1}}{(2 n-2) !} \mathrm{K}_{1}+\frac{(-1)^{n} 3^{n}}{(2 n) !} \\
\quad-\frac{(-1)^{n} 3^{n}}{(2 n+1) !}=0
\end{array}\right.
$$

L'étude de cette suite montre qu'elle est croissante et que la suite $u_{n}=\left(\mathbf{K}_{n} / \mathbf{K}_{n-1}\right)$ est également croissante et tend pour $n=\infty$ vers une limite $r$, qui est la première racine positive de l'équation transcendante:

$$
\begin{aligned}
& \cos \sqrt{\frac{3}{r}}=1-\frac{1}{2 !}\left(\frac{3}{r}\right) \\
& +\frac{1}{4 !}\left(\frac{3}{r}\right)^{2}-\ldots+\frac{(-1)^{n}}{(2 n) !}\left(\frac{3}{r}\right)^{n}+\ldots=0
\end{aligned}
$$

On en déduit :

$$
\sqrt{\frac{3}{r}}=\frac{\pi}{2} \quad r=\frac{12}{\pi^{2}}
$$

La série (2) :

$$
\mathrm{F}^{2}=1+\alpha_{1} \sigma^{2}+\mathrm{K}_{2}\left(\alpha_{1} \sigma^{2}\right)^{2}+\ldots+\mathrm{K}_{n}\left(\alpha_{1} \sigma^{2}\right)^{n}
$$

est done absolument convergente lorsque :

$$
\frac{12 \alpha_{1} \sigma^{2}}{\pi^{2}}<1
$$

DÉTERMiNation DU POTENTIEL DE L'ÉCOULEMENT

Le potentiel de l'écoulement est donné par :

où :

$$
\frac{d z}{d f}=\mathrm{H}\left(f, \sigma^{2}\right)
$$

$$
z=\xi+i \sigma \zeta=\frac{\sigma}{J_{0}}(x+i y) \quad f=\varphi+i \sigma \psi=\frac{\sigma}{V y_{0}}[\Phi+i \Psi]
$$

Les résultats établis plus haut montrent que $\mathrm{H}\left(f, \sigma^{2}\right)$ est donné par (9) :

avec :

$$
\mathrm{H}\left(f, \sigma^{2}\right)=1+\sigma^{2} f_{1}(f)+\sigma^{4} f_{2}(f)+\ldots \sigma^{2 n} f_{n}(f)+\ldots
$$

$$
\begin{gathered}
f_{1}(f)=\frac{\alpha_{1}}{\operatorname{ch}^{2}\left[\left(\sqrt{3 \alpha_{1}} / 2\right) f\right]} \\
f_{2}=a_{21} \alpha_{1} f_{1}+a_{22} f_{1}^{2}=-\alpha_{1} f_{1}+2 f_{1}{ }^{2} \\
f_{n}=a_{n 1} \alpha_{1}^{n-1} f_{1}+\ldots+a_{n p} \alpha_{1}^{n-p} f_{1}{ }^{p}+\ldots+a_{n n} f_{1}{ }^{n}
\end{gathered}
$$

le paramètre $\sigma$ étant relié au nombre de Froude de l'écoulement $F$ par la série (2).

Compte tenu de (19) et (24), on voit que $f_{n}(f)$ est de la forme :

$$
f_{n}(f)=\alpha_{1}{ }^{n} g\left(\sqrt{\alpha_{1}} f\right)=\alpha_{1}{ }^{n} g\left[\sqrt{\alpha_{1}} \sigma\left(\frac{\Phi}{V y_{0}}+i \frac{\Psi}{V y_{0}}\right)\right]
$$

de sorte que $\mathrm{H}\left(f, \sigma^{2}\right)$ et $\mathrm{F}^{2}$ dépendent en fait du seul paramètre $\lambda=\sqrt{\alpha_{1}} \sigma$. Il est donc possible de donner $\dot{a} \alpha_{1}$ une valeur posifive arbitraire. Nous donnerons, dans la suite, à $\alpha_{1}$ la valeur $\alpha_{1}=(4 / 3)$ pour laquelle (19) s'écrit :

$$
f_{1}(f)=\frac{4}{3 \operatorname{ch}^{2} f}
$$


Le potentiel de l'écoulement est alors défini par :

$$
\begin{aligned}
\frac{d z}{d f}=\mathrm{H}\left(\frac{1}{\operatorname{ch}^{2} f}, \sigma^{2}\right)=1 & +\frac{4 \sigma^{2}}{3 \operatorname{ch}^{2} f}+\left(\frac{4 \sigma^{2}}{3}\right)^{2}\left[-\frac{1}{\operatorname{ch}^{2} f}+\frac{2}{\operatorname{ch}^{4} f}\right]+\ldots \\
& +\left(\frac{4}{3} \sigma^{2}\right)^{n}\left[\frac{a_{n 1}}{\operatorname{ch}^{2} f}+\frac{a_{n 2}}{\operatorname{ch}^{4} f}+\ldots+\frac{a_{n n}}{\operatorname{ch}^{2 n} f}\right]+\ldots
\end{aligned}
$$
(2a):

Ce paramètre sans dimensions $\sigma$ étant relié au nombre de Froude de l'écoulement par la séric

$$
\left.\mathrm{F}^{2}=1+\mathrm{K}_{1}(4 / 3) \sigma^{2}+\mathrm{K}_{2}\left[(4 / 3) \sigma^{2}\right]^{2}+\ldots+\mathrm{K}_{n}[4 / 3) \sigma^{2}\right]^{n}+\ldots
$$

absolument convergente pour $|\sigma|<\pi / 4$.

$\mathrm{K}_{1}, \mathrm{~K}_{2} \ldots \mathrm{K}_{n}$ étant donnés par la suite $(29)$, si on pose :

$$
\mathrm{K}_{n}=3^{n} v_{n}, \quad \mathrm{~K}_{1}=3 v_{1}=1,
$$

F² est donné par :

$$
\mathrm{F}^{2}=1+v_{1}\left(4 \sigma^{2}\right)+v_{2}\left(4 \sigma^{2}\right)^{2}+\ldots v_{n}\left(4 \sigma^{2}\right)^{n},
$$

et on observe que la suite $v_{1}=1 / 3, v_{2} \ldots v_{n}$, déduite de (29) par la relation $\mathrm{K}_{n}=3^{n} v_{n}$, est précisément celle qu'on obtient en cherchant les coefficients $d u$ développement $\operatorname{de}(\operatorname{tg} d \sigma) / 2 \sigma$.

On en déduit :

$$
F^{2}=\frac{\operatorname{tg} 2 \sigma}{2 \sigma}
$$

ce qui précise la signification de $\sigma$ : vis-à-vis du nombre de Froude F de l'écoulement, $\sigma$ est la plus petite racine réelle positive de l'équation transcendante (30) où $\mathrm{F}$ est supposé supérieur à 1 .

Ce résultat peut être retrouvé par une autre méthode qui présente un certain intérêt, car elle permet de former des fonctions majorantes, pour la fonction $\mathrm{H}\left[\left(1 / \mathrm{ch}^{2} f\right), \sigma^{2}\right]$ de la variable complexe $f$.

Considérons le potentiel complexe défini par :

$$
\frac{d z}{d f}=\%\left(\frac{1}{\operatorname{ch}^{2} f}, \sigma^{2}\right) \quad \cdot z=\int_{0}^{f} \%\left(\frac{1}{\operatorname{ch}^{2} f}, \sigma^{2}\right) d f
$$

\% étant une fonction holomorphe de $f$, dans toute la région de l'ćcoulement :

$$
[-\infty<0<+\infty \quad 0<4<1]
$$

pour $|\sigma|<\sigma_{0}^{\prime}$, supposons de plus que $\%\left[\left(1 / \operatorname{ch}^{2} f\right), \sigma^{2}\right]$ soit développable en série entière de $\sigma^{2}$ sous une forme analogue à $(9 a)$ :

$$
\frac{d z}{d f}=\chi\left(\frac{1}{\operatorname{ch}^{2} f}, \sigma^{2}\right)=1+\frac{4}{3} \sigma^{2} \frac{a_{11}^{\prime}}{\operatorname{ch}^{2} f}+\ldots+\left(\frac{4}{3} \sigma^{2}\right)^{n}\left[\frac{a_{n 1}^{\prime}}{\operatorname{ch}^{2} f}+\frac{a_{n 2}^{\prime}}{\operatorname{ch}^{4} f}+\ldots+\frac{a_{n n}^{\prime}}{\operatorname{ch}^{2 n} f}\right]+\ldots
$$

cette série est évidemment, pour $|\sigma|<\sigma_{0}^{\prime}$, uniformément convergente, quel que soit $f=\vartheta+i \sigma \psi$, lorsque $-\infty<\varphi<\infty$ et $|\Psi|<1$.

Calculons, comme plus haut, el pour $\psi=1$, la quantité :

$$
\mathrm{Q}\left(\varphi, \sigma^{2}\right)=\frac{2(\zeta-1)\left[\left(\frac{\partial \zeta}{\partial \psi}\right)^{2}+\sigma^{2}\left(\frac{\partial \zeta}{\partial \varphi}\right)^{2}\right]}{\left(\frac{\partial \zeta}{\partial \psi}\right)^{2}+\sigma^{2}\left(\frac{\partial \zeta}{\partial \varphi}\right)^{2}-1}
$$

C'est une fonction de $\varphi$ et de $\sigma^{2}$, donnée par le développement :

$$
\mathrm{Q}\left(\varphi, \sigma^{2}\right)=1+\sigma^{2} \mathrm{U}_{1}(\varphi)+\sigma^{4} \mathrm{U}_{2}(\varphi)+\ldots \sigma^{2 n} \mathrm{U}_{n}(\varphi)+\ldots
$$


uniformément convergent, quel que soit $\varphi$, pour o suffisamment petit, avec :

$$
\begin{aligned}
& \mathrm{U}_{1}(\varphi)=\frac{4}{3} \mathrm{~K}_{1}+\frac{\mathrm{B}_{11}}{\operatorname{ch}^{2} \varphi}=\frac{4}{3}+\frac{2}{\operatorname{ch}^{2} \varphi}\left[\alpha_{11}^{\prime}-1\right] \\
& \mathrm{U}_{2}(\varphi)=\left(\frac{4}{3}\right)^{2} \mathrm{~K}_{2}+\frac{\mathrm{B}_{21}}{\operatorname{ch}^{2} \varphi}+\frac{\mathrm{B}_{22}}{\operatorname{ch}^{4} \varphi} \\
& \mathrm{U}_{n}(\varphi)=\left(\frac{4}{3}\right)^{2} \mathrm{~K}_{n}+\frac{\mathrm{B}_{n 1}}{\operatorname{ch}^{2} \varphi}+\frac{\mathrm{B}_{n 2}}{\operatorname{ch}^{4} \varphi}+\ldots \frac{\mathrm{B}_{n n}}{\operatorname{ch}^{2 n} \varphi}
\end{aligned}
$$

$\mathrm{B}_{n p}$ étant un polynôme de $a_{11}^{\prime}, \ldots \alpha_{(n-1)(n-1)}^{\prime}$ et une fonction linéaire de $\alpha_{n n}^{\prime}, \alpha_{n(n-1)}^{\prime} \ldots a_{n p}^{\prime}$, tous les coefficients $\mathrm{B}_{n p}$ sont identiquement nuls lorsque $a_{n p}^{\prime}=a_{n p}$, quels que soient $n$ et $p$.

On en déduit que $F^{2}$ est égal à la limite de $Q\left(\varphi, \sigma^{2}\right)$ lorsque $\odot$ augmente indéfiniment.

Cette limite étant indépendante des coefficients de (30), il suffit pour la calculer de considérer l'écoulement particulier défini par :

$$
\frac{d z}{d f}=1+\frac{a \sigma^{2}}{\operatorname{ch}^{2} f} \quad z=f+a \sigma^{2} \operatorname{th} f
$$

soit :

d'où :

$$
z=\xi+i \sigma \zeta=\varphi+i \sigma \psi+a \sigma^{2}\left[\frac{\operatorname{sh} 2 \varphi}{\operatorname{ch} 2 \varphi+\cos 2 \sigma \psi}+i \frac{\sin 2 \sigma \psi}{\operatorname{ch} 2 \varphi+\cos 2 \sigma \psi}\right]
$$

$$
\zeta=\psi+\frac{a \sigma \sin 2 \sigma \psi}{\operatorname{ch} 2 \varphi+\cos 2 \sigma \psi}
$$

en calculant $Q\left(\varphi, \sigma^{2}\right)$, en faisant tendre $\varphi$ vers l'infini, on retrouve bien :

$$
\mathrm{F}^{2}=\frac{\operatorname{tg} 2 \sigma}{2 \sigma}
$$

Ceci posé, il est facile de former des développements (30), majorants pour la fonction :

c'est-à-dire tels que $a_{n p}^{\prime}>\left|a_{n p}\right|$.

$$
\mathrm{H}\left(\frac{1}{\operatorname{ch}^{2} f}, \sigma^{2}\right) \text {, }
$$

Considérons, par exemple, la fonction :

$$
\chi\left(\frac{1}{\operatorname{ch}^{2} f}, \sigma^{2}\right)=\frac{1}{1-\frac{\beta \sigma^{2}}{\left[(3 / 4)-\sigma^{2}\right] \operatorname{ch}^{2} f}}
$$

holomorphe, quels que soient $\varphi$ et $\psi[|\psi| \leqslant 1]$ lorsque $|\sigma|<\sigma_{0}^{\prime}$, en désignant par $\sigma_{0}^{\prime}$ la racine réelle positive de l'équation :

$$
\frac{4}{3} \sigma^{2}=\frac{\cos ^{2} \sigma}{\cos ^{2} \sigma+\beta}
$$

sous ces conditions, $\chi\left[\left(1 / \mathrm{ch}^{2} f\right), \sigma^{2}\right]$ est donné par le développement uniformément convergent :

$$
\begin{aligned}
& \frac{1}{1-\frac{\beta \sigma^{2}}{\left[(3 / 4)-\sigma^{2}\right] \operatorname{ch}^{2} f}}=1+\frac{4}{3} \sigma^{2} \frac{\beta}{\operatorname{ch}^{2} f} \\
& \quad+\left(\frac{4}{3} \sigma^{2}\right)^{2} \frac{\beta}{\operatorname{ch}^{2} f}\left(1+\frac{\beta}{\operatorname{ch}^{2} f}\right)+\ldots+\left(\frac{4}{3} \sigma^{2}\right)^{n} \frac{\beta}{\operatorname{ch}^{2} f}\left(1+\frac{\beta}{\operatorname{ch}^{2} f}\right)^{n-1}+\ldots
\end{aligned}
$$

qui est bien de la forme (30).

Ceci posé, on démontre qu'on peut choisir $\beta$ suffisamment grand pour que (31) soit une série majorante du développement $(9 a)$ de $\mathrm{H}\left[\left(1 / \mathrm{ch}^{2} f\right), \sigma^{2}\right]$.

Ơn en conclut que $(9 a)$ est uniformément convergent dans toute la région de l'écoulement pour $|\sigma|<\sigma_{0}, \sigma_{0}$ étant un nombre positif supérieur ou égal au nombre $\sigma_{0}^{\prime}$ défini plus haut. 
L'écoulement est donc défini pour $|\sigma|<\sigma_{0}$ par la série uniformément convergente dans toute la région de l'écoulement:

$$
\begin{aligned}
z=\int_{0}^{f} \mathrm{H}\left(\frac{1}{\operatorname{ch}^{2} f} \sigma^{2}\right) d f=f+ & \frac{4}{3} \sigma^{2} \operatorname{th} f+\left(\frac{4}{3} \sigma^{2}\right)^{2} \operatorname{th} f\left[1-\frac{2}{3} \operatorname{th}^{2} f\right]+\ldots \\
& +\left(\frac{4}{3} \sigma^{2}\right)^{n} \operatorname{th} f\left[b_{n 1}+b_{n 2} \operatorname{th}^{2} f+\ldots+b_{n n} \text { th } 2 n-2 f\right]+\ldots
\end{aligned}
$$

les coefficients' $b_{n 1}, b_{n 2} \ldots b_{n n}$ se déduisant des coefficients $a_{n 1}, a_{n 2} \ldots a_{n n}$ de $(9 a)$ par des relations de récurrence faciles à former.

Rappelons que :

$$
\begin{aligned}
& z=\zeta+i \quad \sigma=\sigma\left(\frac{x+i y}{y_{0}}\right) \\
& f=\varrho+i \sigma \psi=\sigma\left(\frac{\Phi+i \Psi}{V y_{0}}\right)
\end{aligned}
$$

En définitive, l'écoulement envisagé est défini par la fonction analytique :

dans laquelle :

$$
\frac{x+i y}{y_{0}}=\frac{\Phi+i \Psi}{V y_{0}}+\frac{1}{\sigma} \mathbf{G}\left(\operatorname{th} f, \sigma^{2}\right)
$$

$$
f=\sigma\left(\frac{\Phi+i \Psi}{\mathrm{V} y_{0}}\right)
$$

$\sigma$ est un paramètre sans dimensions relié au nombre de Froude $F$ de l'écoulement par la relation $(30)$ :

$$
\mathrm{F}^{2}=\frac{\operatorname{tg} 2 \sigma}{2 \sigma}
$$

G (th $\left.f, \tau^{2}\right)$ est une fonction holomorphe de $f$ dans toute la région de l'ćcoulement lorsque $|\sigma|<\sigma_{0}$, donnée par le développement.

$$
\begin{aligned}
\mathrm{G}\left(\operatorname{th} f, \sigma^{2}\right)=\frac{4}{3} \sigma^{2} \text { th } f+\left(\frac{4}{3} \sigma^{2}\right)^{2} \text { th } f & \left(1-\frac{2}{3} \operatorname{th}^{2} f\right)+\ldots \\
& +\left(\frac{4}{3} \sigma^{2}\right)^{n}\left[b_{n 1}+b_{n 2} \operatorname{th}^{2} f+\ldots+b_{n n} \text { th } 2 n-2 f\right]+\ldots
\end{aligned}
$$

Les lignes de courant, et en particulier la surface libre $(\psi=1)$, s'obtiennent aisément en séparant dans (32) les parties réelles et imaginaires.

On a :

d'où :

$$
\text { th } f=a+b i=\frac{\operatorname{sh} 2 \varphi}{\operatorname{ch} 2 \varphi+\cos 2 \sigma \psi}+i \frac{\sin 2 \sigma \psi}{\operatorname{ch} 2 \varphi+\cos 2 \sigma \psi}
$$

$$
\begin{aligned}
& \xi=\frac{x}{y_{0}}=\frac{\varphi}{\sigma}+\frac{4}{3} \sigma a+\left(\frac{4}{3}\right)^{2} \sigma^{3} \frac{a}{3}\left[3\left(1+2 b^{2}\right)-2 a^{2}\right]+\ldots \\
& \zeta=\frac{y}{y_{0}}=\psi+\frac{4}{3} \sigma b+\left(\frac{4}{3}\right)^{2} \sigma^{3} \frac{b}{3}\left[3\left(1-2 a^{2}\right)+2 b^{2}\right]+\ldots
\end{aligned}
$$

expressions dans lesquelles :

$$
a=\frac{\operatorname{sh} 2 \varphi}{\operatorname{ch} 2 \varphi+\cos 2 \sigma \psi} \quad b=\frac{\sin 2 \sigma \psi}{\operatorname{ch} 2 \varphi+\cos 2 \sigma \psi}
$$

Les composantes de la vitesse s'obtiendraient de même en séparant dans ( $9 a$ ) les parties réelles et imaginaires.

Il reste, pour être complet, à déterminer le coefficient du terme en $\sigma^{6}$ dans les développements $(9 a)$ et $(32)$ et à préciser la valeur $\sigma_{0}$, pour laquelle apparaît une singularité dans' l'écoulement (onde solitaire limite). 
Le calcul est pénible, mais ne soulève pas de difficultés, la forme générale de $f_{n}$ (ஒ) ayant èté précisée.

On obtient, au quatrième ordre près :

$$
\begin{aligned}
\frac{d z}{d f}=1+\frac{4}{3} \sigma^{2} \frac{1}{\operatorname{ch}^{2} f}+\left(\frac{4}{3} \sigma^{2}\right)^{2} & {\left[-\frac{1}{\operatorname{ch}^{2} f}+\frac{2}{\operatorname{ch}^{4} f}\right] } \\
& +\left(\frac{4}{3} \sigma^{2}\right)^{3}\left[\begin{array}{c}
2 \\
15 \operatorname{ch}^{2} f
\end{array}-\frac{58}{15 \operatorname{ch}^{4} f}+\frac{68}{15 \operatorname{ch}^{6} f}\right]+\ldots \\
z=f+\frac{4}{3} \sigma^{2} \operatorname{th} f+\left(\frac{4}{3} \sigma^{2}\right)^{2} \operatorname{th} f & {\left[1-\frac{2}{3} \operatorname{th}^{2} f\right] } \\
& +\left(\frac{4}{3} \sigma^{2}\right)^{3} \operatorname{th~} f\left[\frac{12}{15}-\frac{26}{15} \operatorname{th}^{2} f+\frac{68}{75} \operatorname{th}^{4} f\right]+\ldots
\end{aligned}
$$

La valeur de l'ordonnée $y_{c}$ à la crête s'obtient en faisant dans (34) $\varphi=0 \quad \psi=1$. Il vient :

$$
\begin{aligned}
\zeta_{0}=\frac{y_{c}}{y_{0}}=1+\frac{4}{3} \sigma^{2} \times \frac{\operatorname{tg} \sigma}{\sigma}+ & \left(\frac{4}{3} \sigma^{2}\right)^{2} \frac{\operatorname{tg} \sigma}{\sigma}\left(1+\frac{2}{3} \operatorname{tg}^{2} \sigma\right) \\
& +\left(\frac{4}{3} \sigma^{2}\right)^{3} \frac{\operatorname{tg} \sigma}{\sigma}\left[\frac{12}{15}+\frac{26}{15} \operatorname{tg}^{2} \sigma+\frac{68}{75} \operatorname{tg}^{4} \sigma\right]+\ldots
\end{aligned}
$$

\section{Calcul DE $\sigma_{0}$}

$\sigma_{0}$ est la valeur de $\sigma$ pour laquelle apparaît une singularité dans l'écoulement, la vitesse s'annulant à la crête (dans l'écoulement permanent envisagé). Le point $\varphi=0 \quad \psi=1$ est donc un point singulier pour la fonction $\mathrm{G}\left(\right.$ th $\left.f, \sigma_{0}^{2}\right)$.

Srokes a montré que la nature de ce point singulier, pour une houle irrotationnelle quelconque, est imposée par la condition à la surface libre. C'est un point de ramification correspondant à un radical arithmétique de puissance $1 / 3$.

Il s'ensuit que pour $\varphi==0, \psi=1, \sigma=\sigma_{0}$, la série (9a) donnant $d z_{\prime}^{\prime} d f$ est divergente (*), la sé rie (35) étant cependant convergente.

Ecrivons la condition à la surface libre, à la crête, pour l'onde limite $(u=0, v=0$ pour $\stackrel{\varphi}{=0} \quad \psi=1$ ), il vient :

$$
2\left(\zeta_{c}-1\right)=\mathrm{F}^{2}=\frac{\operatorname{tg} 2 \sigma}{2 \sigma} .
$$

$\sigma_{0}$ est donc la première racine positive de l'équation transcendante (36), dans laquelle le second membre est donné sous la forme d'une série:

$$
\begin{aligned}
\frac{\operatorname{tg} 2 \sigma}{4}=\frac{4}{3} \sigma^{2} \operatorname{tg} \sigma+\left(\frac{4}{3} \sigma^{2}\right)^{2} \operatorname{tg} \sigma & \left(1+\frac{2}{3} \operatorname{tg}^{2} \sigma\right) \\
& +\left(\frac{4}{3} \sigma^{2}\right)^{3} \operatorname{tg} \sigma\left(\frac{12}{15}+\frac{26}{15} \operatorname{tg}^{2} \sigma+\frac{68}{75} \operatorname{tg}^{4} \sigma\right)+\ldots
\end{aligned}
$$

malheureusement, comme on ne connait que les trois premiers termes de cette série, on obtient, en résolvant (36), une valeur approchée par excès. L'approximation obtenue sera meilleure si l'on peut déterminer une valeur approchée du reste de la série, constituée par le seeond membre de (36).

Cette série s'écrit:

$$
u_{1}(\sigma)+u_{2}(\sigma)+u_{3}(\sigma)+\ldots+u_{n}(\sigma)+\ldots
$$

$\left.{ }^{*}\right)$ D'ailleurs $(d z / d f)=\left(\mathrm{V}(u+i b) / u^{2}+b^{2}\right.$ n'est pas défini pour $u=0 \quad n=0$. 
avec :

$$
u_{n}(\sigma)=\left(\frac{4}{3} \sigma^{2}\right)^{n} \operatorname{tg} \sigma\left[b_{n 1}-b_{n 2} \operatorname{tg}^{2} \sigma+\ldots+(-1)^{n+1} b_{n n} \operatorname{tg}{ }^{2 n-2} \sigma\right],
$$

$b_{n 1}, b_{n 2} \ldots b_{n n}$ étant les coefficients du terme général d'ordre $n$ de (32). Cette série à termes positifs est uniformément convergente pour $\sigma \leqslant \sigma_{0}$; la suite $r_{n}(\sigma)=\left[u_{n}(\sigma) / u_{n-1}(\sigma)\right]$ tend donc, lorsque $n$ augmente indéfiniment, vers une limite $r(\sigma)$ inférieure à 1 , quel que soit $\sigma$.

Admettons maintenant, ce qui est vérifié pour les deux premiers termes, que les deux suites $r_{n}(\sigma)$ et $\rho_{n}(\sigma)=2 r_{n}-r_{n-1}$ constituent deux ensembles adjacents ayant pour borne commune $r(\sigma)$, et désignons par :

$$
\mathrm{R}_{n}(\sigma)=u_{n}(\sigma)+u_{(n+1)} \sigma+\ldots
$$

le reste de la série, on a :

$$
u_{(n-1)}(\sigma) \frac{r_{n-1}(\sigma)}{1-r_{n-1}(\sigma)}<\mathrm{R}_{n}(\sigma)<u_{(n-1)}(\sigma) \frac{\rho_{n-1}(\sigma)}{1-\rho_{n-1}(\sigma)}
$$

en appliquant ce résultat au calcul de $R_{4}(\sigma)$ et en remplaçant, dans $(36), R_{4}(\sigma)$ par ses valeurs approchées par défaut et par excès, on voit que :

$$
0,54625<\sigma_{0}<0,54654,
$$

ce qui donne la valeur de $\sigma_{0}$ avec trois décimales exactes.

Le nombre de Froude $\mathbf{F}_{0}$, correspondant à l'onde solitaire limite, est donné par :

$$
F_{0}^{2}=\frac{\operatorname{tg} 2 \sigma_{0}}{2 \sigma_{0}}
$$

on a :

$$
1,76558<\mathrm{F}_{0}{ }^{2}<1,76707
$$

d'où $\mathrm{F}_{0}{ }^{2}=1,766$ avec trois décimales exactes; la profondeur d'eau limite à la crête s'en déduit

par :

$$
\frac{\underline{y}_{c}}{y_{0}}=\zeta_{\mathrm{r}}=1+\frac{\mathrm{F}_{0}{ }^{2}}{2}=\underline{\underline{1,883}}
$$

La valeur de $\zeta_{c}$, déduite de (35) [avec $\sigma=\sigma_{0}$ ], donnerail pour la quatrième approximation, c'estì-dire en négligeant le reste de la série :

$$
\zeta_{c}=1,772
$$

ce qui correspond à une approximation de $6 \%$. L'approximation obtenue serait évidemment hien meilleure pour $\sigma<\sigma_{0}$.

Pour terminer, il est intéressant de comparer cette théorie avec celle classique de Boussineso, ainsi qu'avec les résultats récemment exposćs dans la Houille Blanche par M. J. N. Hun'T.

Pour obtenir l'approximation de Boussinese, considérons les deux premiers termes des équations $(32 a)$, qui s'écrivent :

$$
\begin{gathered}
\frac{\xi}{\sigma}=\frac{x}{y_{0}}=\frac{\varphi}{\sigma}+\frac{4}{3} \sigma a \\
\zeta=\frac{y}{y_{0}}=\psi+\frac{4}{3} \sigma b
\end{gathered}
$$

avec :

$$
a=\frac{\operatorname{sh} 2 \varphi}{\operatorname{ch} 2+\cos 2 \sigma \psi} \quad b=\frac{\sin 2 \sigma \psi}{\operatorname{ch} 2 \%+\cos 2 \sigma \psi}
$$

et développons $a$ et $b$ en série entière de $\psi$, en négligeant les termes en $\psi^{2}$. Il vient:

$$
\left\{\begin{array}{c}
\frac{\xi}{\sigma}=\frac{x}{y_{0}}=\frac{\varphi}{\sigma}+\frac{4}{3} \sigma \text { th } \varphi \\
\zeta=\frac{y}{y_{0}}=\psi+\frac{4}{3} \sigma^{2} \frac{\psi}{\operatorname{ch}^{2} ?}
\end{array}\right.
$$


On retrouverait d'ailleurs les mêmes résultals en considérant les deux premiers termes des équations (10) el (11) qui constituent en fait des séries doubles en a et $\psi$, dont la convergence est lente, ce qui n'est pas le cas de (34).

On a de même, pour la célérité, en prenant les deux premiers termes de (2):

$$
\mathrm{F}^{2}=1+\frac{4}{3} \sigma^{2}
$$

L'équation de la surface libre s'obtient alors en éliminant entre les équations (37) et, en faisant $\downarrow=1$ à l'approximation envisagée, il vient :

$$
\frac{y}{y_{0}}=1+\frac{4}{3} \sigma^{2} \frac{1}{\operatorname{ch}^{2} \sigma\left(x / y_{0}\right)}
$$

avec :

$$
\sigma=\frac{\sqrt{3}}{2} \sqrt{\mathbf{F}^{2}-1}
$$

qui correspond à la solution de BoussinesQ.

On remarque, par l'examen de (11) et (12), qu'au premier ordre, la composante horizontale $u$ de la vitesse peut être considérée comme uniformément répartie dans une section, la composante verticale ayant une répartition triangulaire.
La répartition donnée par Boussinese ćlait parabolique, alor's qu'en fait elle l'est seulement si on considère les termes du second ordre, ce qui exige la détermination de $f_{2}(\varphi)$.

En définitive, la solution de Boussineso ne constitue pas une première approximation de l'écoulement et du potentiel complexe, mais un développement de cetle première approximation par rapport à la fonction de courant $\psi$, développement limité au premier terme.

La solution développée par J.N. Hunt est constituée également par un développement en série double de $\sigma$ et $\psi$ limité au troisième terme, l'auteur s'étant d'ailleur's attaché uniquement à la détermination de la célérité de la propagation et à l'équation de la surface libre $[\psi=1]$.

Ce développement s'obtient, de la même façon, à l'aide des équations (10) et (11) limitées en terme en $\sigma^{6}$, ce qui nécessite, comme nous l'avons fait, la détermination des fonctions $f_{1}(\varphi), f_{2}(\varphi)$ et $f_{3}(\varphi)$. En faisant $\psi==1$, la surface libre est déterminée paramétriquement en fonction de $\varphi$.

On obtiendrait ainsi des développements que nous n'écrirons pas, mais qui diffèrent, pour les deuxième et troisième approximations, de ceux établis par J. N. Hunr, par la présentation, dans ces derniers, de termes de la forme o sh $0 / \mathrm{ch}^{3} \%$ (dans les deuxic̀me et troisième approximations), et $\rho^{2} \operatorname{sh}^{2} \rho / \mathrm{ch}^{4} \varphi$ (dans la troisième approximation).

On en conclut, ainsi d'ailleurs que nous l'avons établi plus haut (détermination du coefficient de $\sigma^{4}$ ), que la série déterminant la surface libre, dans l'étude de M. Hunt, n'est pas uniformément convergente, quel que soit $\varphi$.

En revanche, l'expression, donnée par l'auteur, de la célérité de propagation est exacte, à l'approximation envisagée.

On obtient, au troisième ordre, à partir de (35) et (2 a) :

$$
\begin{gathered}
\frac{h}{y_{0}}=\zeta_{\rho}-1=\frac{4}{3} \sigma^{2} \frac{\operatorname{tg} \sigma}{\sigma}+\left(\frac{4}{3} \sigma^{2}\right)^{2} \frac{\operatorname{tg} \sigma}{\sigma}\left(1+\frac{2}{3} \operatorname{tg}^{2} \sigma\right) \\
\mathrm{F}^{2}=\frac{\operatorname{tg}^{2} \sigma}{2 \sigma}=1+\frac{4 \sigma^{2}}{3}+\frac{12}{10}\left(\frac{4 \sigma^{2}}{3}\right)^{2}+\frac{\operatorname{tg} \sigma}{\sigma}\left[\frac{12}{15}+\frac{26}{15} \operatorname{tg}^{2} \sigma+\frac{68}{75} \operatorname{tg}^{4} \sigma\right] \\
3
\end{gathered}
$$

en éliminant $\sigma$ entre ces deux équations, on retrouve bien, au quatrième ordre près, l'expression obtenue par M. J. N'. HunT:

$$
\mathrm{F}^{2}=1+\frac{h}{y_{0}}-\frac{1}{20}\left(\frac{h}{y_{0}}\right)^{2}-\frac{3}{70}\left(\frac{h}{y_{0}}\right)^{3}
$$

